\title{
CBIR using Textural Feature
}

\author{
Nilam N Ghuge \\ Research Scholar (JJT UNIV.) \\ Assistant Professor, Electrical \\ B.S.I.O.T.R.(W), Pune (India)
}

\author{
Parul S Arora Bhalotra \\ Research Scholar (JJT Univ.) \\ Assistant Professor, ETC \\ G.H.R.C.E.M. Pune (India)
}

\author{
B. D. Shinde, PhD. \\ Professor, ETC \\ G.N.V.O.I.T., Tala \\ Raigarh (India)
}

\begin{abstract}
CBIR system focuses on retrieving images from the database; the system depends on the way the indexing is being implemented. The way or method in which an image is stored will affect how it will be retrieved later and which can save more storage space and improve the retrieval process. Building effective content-based image retrieval (CBIR) systems involves the combination of image creation, storage, security, transmission, analysis, evaluation feature extraction, and feature combination in order to store and retrieve images effectively.

The goal of CBIR systems is to support image retrieval based on content i.e. shape, color, texture. In this paper we have implemented CBIR techniques using conventional Histogram and Gabor filter. We have shown results of query image and retrieved image also 2D frequency response of Gabor filter with various angles as it is direction dependent filter. We have used Euclidean distance as a measure to calculate distance between two images.
\end{abstract}

\section{General Terms}

Hybrid Intelligent Systems for Computer Vision and Pattern Recognition, Image Processing, Texture features, CBIR, Filter.

\section{Keywords}

Content based Image retrieval, Histogram, Gabor function, Euclidean distance, Precision, Recall.

\section{INTRODUCTION}

With the rapid proliferation of the internet and the worldwideweb, the amount of digital image data accessible to users has grown enormously. Image databases are becoming larger and more widespread, and there is a growing need for effective and efficient image retrieval (IR) systems.

All the information available is only useful if one can access it efficiently. This does not only mean fast access from a storage management point of view but also means that one should be able to find the desired information without scanning all information manually.

An important part of digital media is image data. In contrast to text, images just consist of pure pixel data with no inherent meaning. Commercial image catalogues therefore use manual annotation and rely on text retrieval techniques for searching particular images. However, such an annotation has two main drawbacks:

First, the annotation depends on the person who adds it. Naturally the result may vary from person to person and furthermore may depend on the context. Within a general image database it may be sufficient to just add an annotation like "butterfly" whereas this obviously is not sufficient for a biologist's database consisting of different types of butterflies only.

The second problem with manual annotation is that it is very time consuming. While it may be worthwhile for commercial image collections, it is prohibitive for indexing of images within the World Wide Web. One could not even keep up with the growth of available image data.

So we need a system which can effectively retrieve the desired image even if the database is not annotated. Imaging is a major factor in areas such as art galleries, interior design and weather forecasting. It is important for those areas to be able to retrieve the stored image quickly and accurately. The more effective the images are being stored, the more efficient the images can be retrieved later; this is where Content-Based Image Retrieval (CBIR) indexing comes in. Several existing applications, such as Query By Image (QBIC) which handles image databases and allows user to insert queries or interact with provided interfaces have focus on CBIR. Some of the applications have even used new algorithms or methods that help bring better result in retrieval process. However, there is potential to improve the existing algorithms, which increases the effectiveness of the retrieval process. Those existing algorithms have their own advantages and disadvantages, but we can use them by trying to combine and come up with a new algorithm that reduces the limitation of existing algorithms [1].

CBIR system focuses on retrieving images from the database; the system depends on the way the indexing is being implemented. The way or method in which an image is stored will affect how it will be retrieved later. This work aim is to develop an indexing algorithm based on existing CBIR studies, which can save more storage space and improve the retrieval process.

Image retrieval has become more significant when people start to have large collection of images which they need to use at some point. The idea of searching those collections one by one to match manually with what the user wants (user's query) is a nightmare. This is where Content-Based Image Retrieval (CBIR) comes in to solve the problem. CBIR has come long way before 1990 and very little papers has been published at that time, however the number of papers published since 1997 is increasing. This indicated that more people have become interested with this area of research.

There are many CBIR algorithms as the result of those researches and most of those algorithms process image into several layers of tasks. Those layers of tasks consist of extracting the multidimensional features of an image query and compare it with images in the database are perform after the system populate database with images [2]. Populating database with extracted information from the images and indexed appropriately will affect the performance of retrieval 
[1]. The information consists of color, shape, texture and the rest of image's characteristic.

Features that most method focuses on are color, shape and texture. For color, a significant improvement over the RGBcolor space use of opponent color representation uses the opponent color axes (R-G, 2B-R-G, R+G+B) is one way to represent color image. There is another method called Color Predominance Method which scans the image and replaces each pixel color with the new RGB color list[10].

Users tend to use three kinds of research strategies. One of them is search by association when they have no specific aim other than find interesting things. Another class of users aims to search for a specific image. Users may have a specific image in mind and the target is interactively specified as similar to a group of given examples, which are useful for art, industrial components or catalogues. The third class of applications may be the category search. Users aim to retrieve an image from a specific class.

\section{CBIR USING COLOR HISTOGRAM}

Comparing two images and deciding if they are similar or not is a relatively easy thing to do for a human. Getting a computer to do the same thing effectively is however a different matter. Many different approaches to CBIR have been tried and many of these have one thing in common, the use of colour histograms.

For content based image retrieval to work, we have to find some features of the image that can be used when comparing it with another. One of the features most popular for image indexing and retrieval is color. Comparing the colour distribution of two images will often say something about their similarity.

Comparing all the colors in two images would however be very time consuming and complex, and so a method of reducing the amount of information must be used. One way of doing this is by quantizing the color distribution into color histograms. First introduced by Swain and Ballard [3], and used by many others, this is probably one of the more popular approaches to image retrieval today.

The color histogram for an image is constructed by counting the number of pixels of each colour.

When computing a color histogram for an image, the different color axes are divided into a number called bins. A three dimensional $8 \times 8 \times 8$ RGB histogram would therefore contain a total of 512 such bins. When indexing the image, the color of each pixel is found, and the corresponding bin's count is incremented by one [4].

An image histogram refers to the probability mass function of the image intensities. This is extended for color images to capture the joint probabilities of the intensities of the three colour channels. More formally, the color histogram is defined by,

$$
\mathrm{h}_{\mathrm{A}, \mathrm{B}, \mathrm{C}}(\mathrm{a}, \mathrm{b}, \mathrm{c})=\mathrm{N} \cdot \operatorname{Prob}(\mathrm{A}=\mathrm{a}, \mathrm{B}=\mathrm{b}, \mathrm{C}=\mathrm{c})
$$

where A, B and C represent the three color channels (R,G,B or $\mathrm{H}, \mathrm{S}, \mathrm{V}$ ) and $\mathrm{N}$ is the number of pixels in the image. Computationally, the colour histogram is formed by discretizing the colours within an image and counting the number of pixels of each color.

There are several distance formulas for measuring the similarity of color histograms. Three distance formulas that have been used for image retrieval including histogram Euclidean distance, histogram intersection and histogram quadratic (cross) distance [5, 6].

In this work we had calculate Histogram Euclidean distance : Let $\mathrm{h}$ and $\mathrm{g}$ represent two color histograms. The Euclidean distance between the color histograms $\mathrm{h}$ and $\mathrm{g}$ can be computed as:

$\mathrm{d}^{2}(\mathrm{~h}, \mathrm{~g})=\sum_{\mathrm{A}} \sum_{\mathrm{B}} \sum_{\mathrm{C}}(\mathrm{h}(\mathrm{a}, \mathrm{b}, \mathrm{c})-\mathrm{g}(\mathrm{a}, \mathrm{b}, \mathrm{c}))^{2}$

In this distance formula, there is only comparison between the identical bins in the respective histograms. Two different bins may represent perceptually similar colors but are not compared crosswise. All bins contribute equally to the distance. The minimum distance value signifies an exact match with the query. Figure 1 shows database used. Figure 2 shows query image and retrieved images. Figure 3 shows corresponding histogram of query and retrieved images.

\section{CBIR USING GABOR FILTER}

The Gabor filter has been widely used to extract image features, especially texture features [7,8]. It is optimal in terms of minimizing the joint uncertainty in space and frequency, and is often used as an orientation and scale tunable edge and line (bar) detector. As the Gabor filters are direction dependent, the Gabor transform of an image is to be performed for all chosen directions. Thus then set of angles used in Gabor feature extraction does affect the results in applications such as Content Based Image Retrieval (CBIR).

The basic idea of using Gabor filters to extract texture features is as follows.

A two dimensional Gabor function $\mathrm{g}(\mathrm{x}, \mathrm{y})$ is defined as:

$g(x, y)=\frac{1}{2 \pi \sigma_{x} \sigma_{y}} \exp \left[-\frac{1}{2}\left(\frac{x^{2}}{\sigma_{x}^{2}}+\frac{y^{2}}{\sigma_{y}^{2}}\right)+2 \pi j W x\right]$

where, $\sigma_{\mathrm{x}}$ and $\sigma_{\mathrm{y}}$ are the standard deviations of the Gaussian envelopes along the $\mathrm{x}$ and $\mathrm{y}$ direction.

Then a set of Gabor filters can be obtained by appropriate dilations and rotations of $\mathrm{g}(\mathrm{x}, \mathrm{y})$ :

$$
\begin{aligned}
& g_{m n}(x, y)=a^{-m} g\left(x^{\prime}, y^{\prime}\right) \\
& x^{\prime}=a^{-m}(x \cos \theta+y \sin \theta) \\
& y^{\prime}=a^{-m}(-x \sin \theta+y \cos \theta)
\end{aligned}
$$

Where $\mathrm{a}>1, \theta=\frac{\mathrm{n} \pi}{\mathrm{k}} \quad \mathrm{n}=0,1, \ldots \ldots \ldots, \mathrm{K}-1$ and $\mathrm{m}=$ $0,1, \ldots \ldots, \mathrm{S}-1 . \mathrm{K}$ and $\mathrm{S}$ are the number of orientations and scales. The scale factor $\mathrm{a}^{-\mathrm{m}}$ is to ensure that energy is independent of $\mathrm{m}$.

Given an image $\mathrm{I}(\mathrm{x}, \mathrm{y})$, its Gabor transform is defined as:

$\mathrm{w}_{\mathrm{mn}}(\mathrm{x}, \mathrm{y})=\int \mathrm{I}(\mathrm{x}, \mathrm{y}) \mathrm{g}_{\mathrm{mn}}^{*}\left(\mathrm{x}-\mathrm{x}_{1}, \mathrm{y}-\mathrm{y}_{1}\right) \mathrm{dx}_{1} d \mathrm{y}_{1}$

Where $*$ indicates complex conjugate. Then the mean $\mu_{\mathrm{mn}}$ and the standard deviation $\sigma_{\mathrm{mn}}$ of the magnitude of $\mathrm{W}_{\mathrm{mn}}(\mathrm{x}, \mathrm{y})$ can be used to represent the texture feature of a homogenous texture region. Substitute angle $\theta$ by $0^{0}, 45^{\circ}, 90^{\circ}, 135^{\circ}, 180^{\circ}$ respectively.

After applying the Gabor Filter, the texture features of images are extracted by using mean function. For retrieving the images from the dataset, the Distance Metric Measures for every images are calculated. The minimum distance value signifies an exact match with the query. Gabor transform has selectivity for orientation, spectral bandwidth and spatial extent [9].

Figure 4 shows query image and retrieved image using Gabor filter. Figure 5 shows energy of query and retrieved image. Figure 6 shows filtered image with $0^{0}$ angle, Figure 7 shows 2D frequency response of Gabor filter with $0^{0}$ for first retrieved image. Figure 8 shows filtered image with $45^{\circ}$ angle, Figure 9 shows 2D frequency response of Gabor filter with $45^{0}$ for first retrieved image. Figure 10 shows filtered image with $90^{\circ}$ angle, Figure 11 shows 2D frequency response of Gabor filter with $90^{\circ}$ for first retrieved image. Figure 12 shows filtered image with $135^{\circ}$ angle, Figure 13 shows 2D 
frequency response of Gabor filter with $135^{0}$ for first retrieved image.

\section{RESULTS AND DISCUSSION}

The performance or evaluation of the image retrieval algorithm is measured by Precision and Recall curve. $[11,12]$

Precision $=\frac{\text { Number of relevant images retrieved }}{\text { Total number of images retrieved }}$

Recall $=\frac{\text { Number of relevant images retrieved }}{\text { Total number of revalent images }}$

We have computed precision - recall values for queries. Figure 14 and Figure 15 shows Histogram based retrieval effectiveness and Gabor Filter based retrieval effectiveness.

\section{DATABASE}
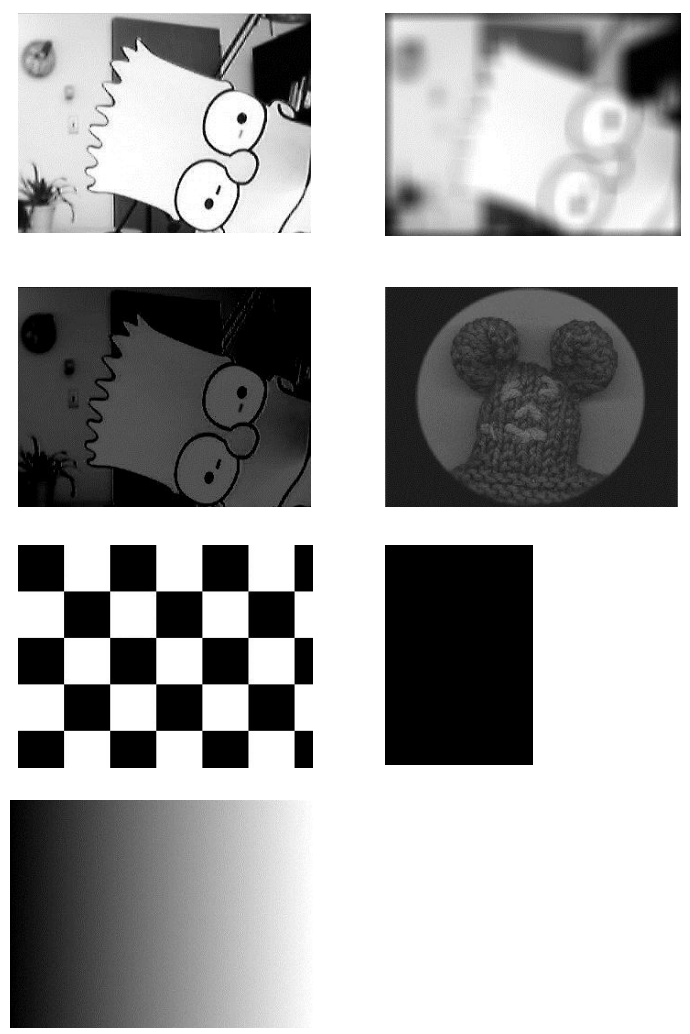

Figure 1

Query image and retrieved images
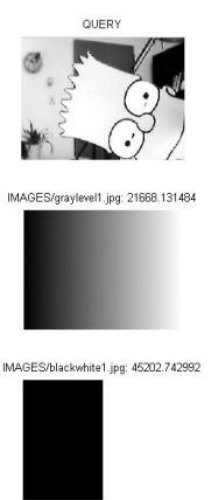
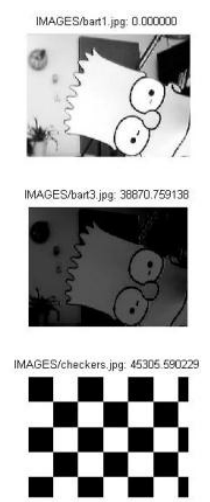
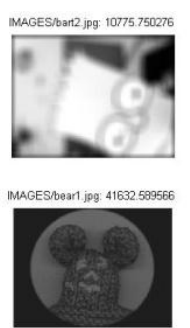

Figure 2

Histogram of query and retrieved images
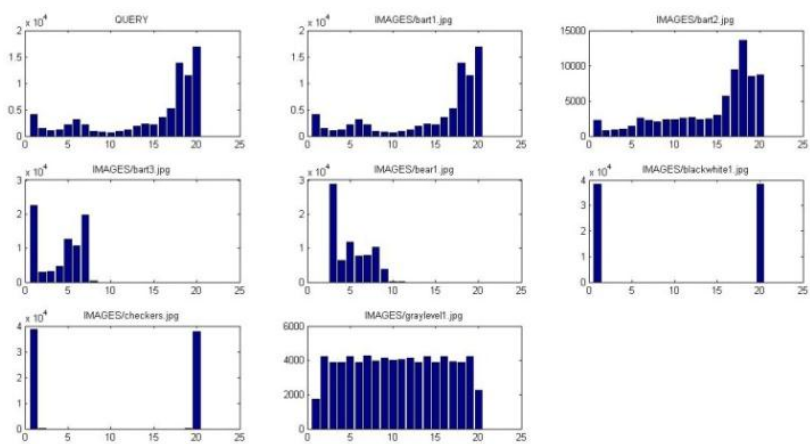

Figure 3

Query image and retrieved image using Gabor filter.
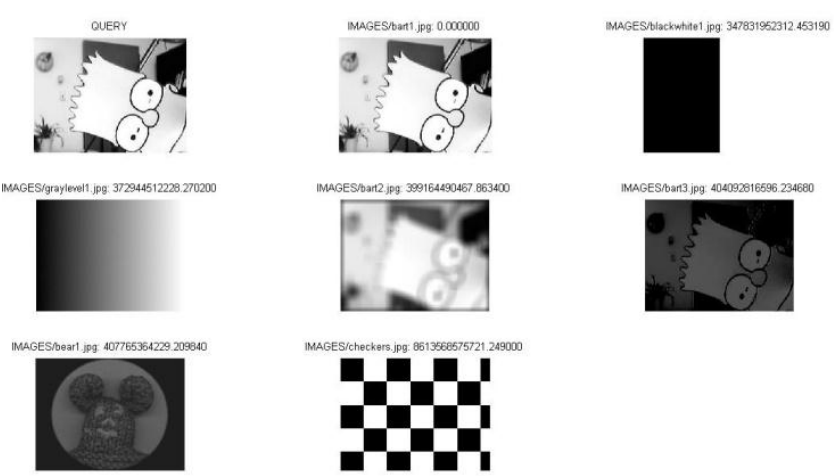

Figure 4

Energy of query and retrieved image
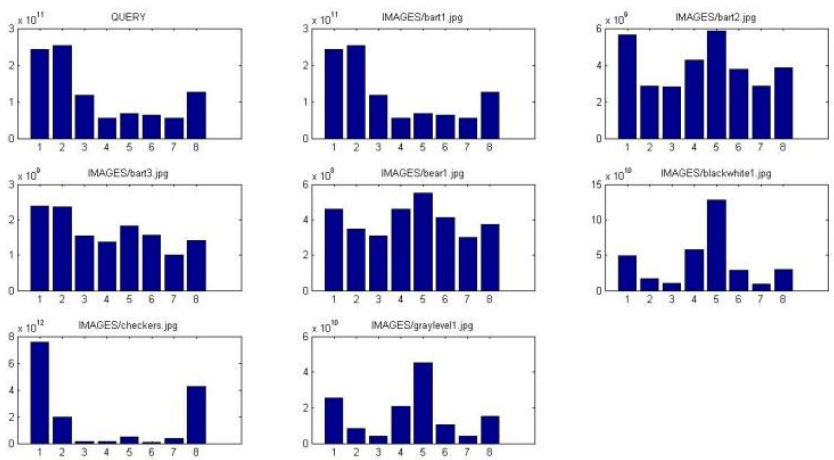

Figure 5

Filtered image \& 2D frequency response of Gabor filter with $0^{0}$ angle

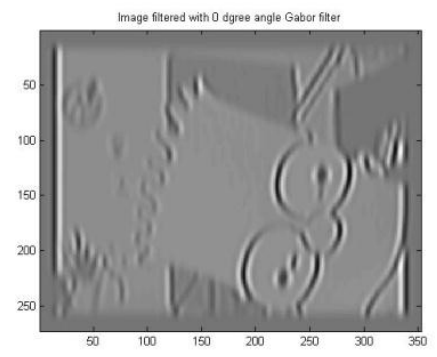

Figure 6

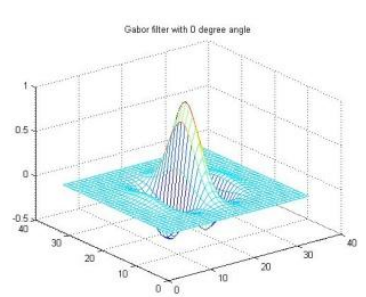

Figure 7 
Filtered image \& 2D frequency response of Gabor filter with $45^{\circ}$ angle.

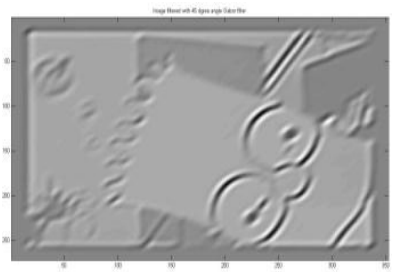

Figure 8

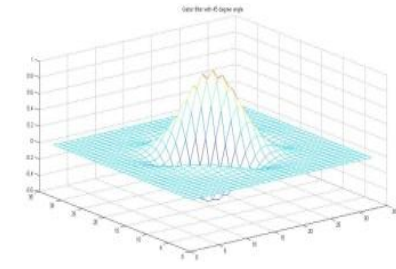

Figure 9
Filtered image \& 2D frequency response of Gabor filter with $90^{\circ}$ angle.

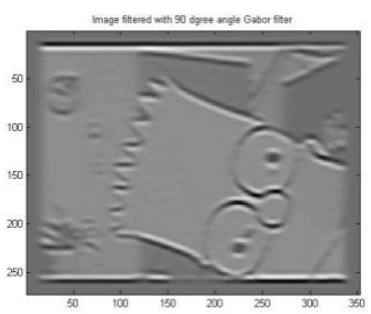

Figure 10

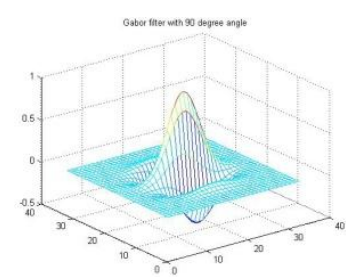

Figure 11
Filtered image \& 2D frequency response of Gabor filter with $135^{\circ}$ angle.

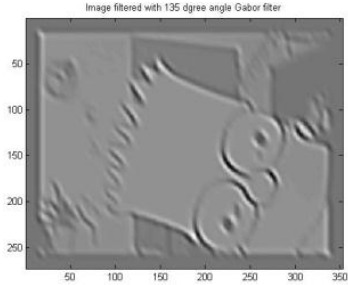

Figure 12

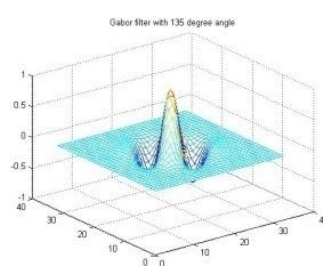

Figure 13
Histogram based retrieval effectiveness

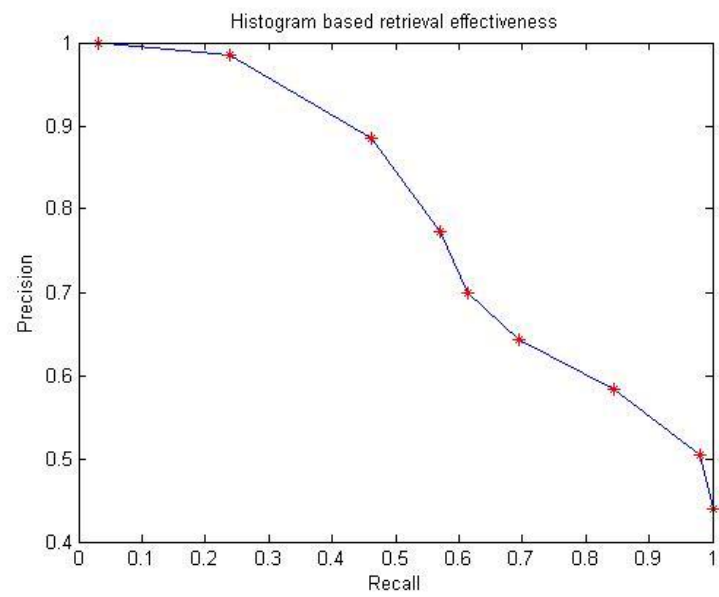

Figure 14

\section{Gabor Filter based retrieval effectiveness}

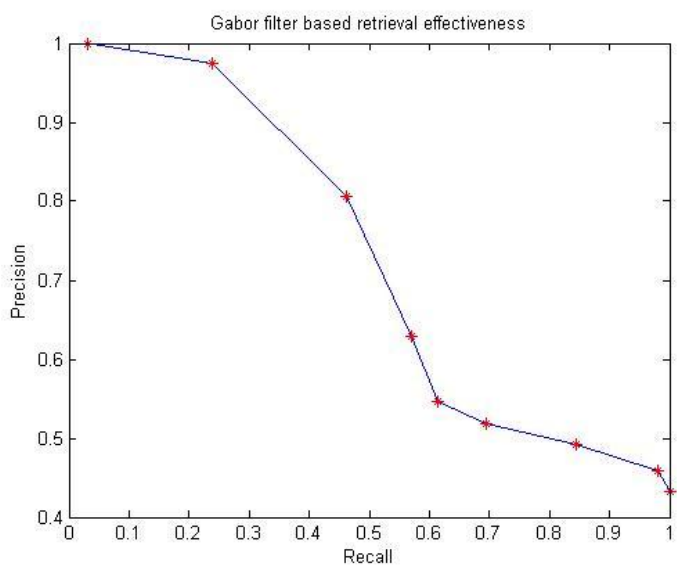

Figure 15

\section{CONCLUSION}

In this paper, we have described the Histogram and Gabor filter based method for image retrieval, a comparative study has been carried out on feature extraction techniques and extracts the feature of texture, Color of color image with Euclidean distance metrics measures are taken for retrieving the similar images from the data set. From this experiment more images are retrieved by using Gabor than Color Histogram technique. Also we have calculated 2D response of the Gabor filter with filtered image. Therefore to provide better performance of retrieving more images are needed on the basis of these techniques. The future direction of the research can be extended based on the same lines. Similar to gabor filter Wavelet transform and its extension Complex wavelet transform does extract the directional information from images. The extra advantage of using wavelet transform would be analyzing the image features at various resolutions. We plan to extend the research in similar direction to analyze the performance of the system using multiresolution features.

\section{REFERENCES}

[1] R. Datta, D. Joshi, J. Li \& J. Z. Wang, April 2008, "Image retrieval: Ideas, inuences \& trends of the new age”, ACM Computer surv., vol. 40, no.2, pp. 160-163.

[2] M. S. Lew, N. Sebe, C. Djeraba, and R. Jain, February 2006, "Content-based multimedia information retrieval: State of the art and challenges", ACM Trans. multimedia computer communication. Appl., vol. 2, no. 1, pp. 1-19

[3] James Z. Wang, "Integrated Region-Based Image Retrieval", Boston, Kluwer Academic Publishers,2001

[4] P.S.Suhasini ,Dr. K.SRI Rama Krishna, Dr. I. V. Muralikrushna,2008, "CBIR using color histogram processing", Journal of theoretical and applied information technology, vol. 6, no.1, pp. 116-122

[5] J. R. Smith and S.-F. Chang. " Automated image retrieval using color and texture", Technical Report CU/CTR 40895-14, Columbia University, July 1995.

[6] J. R. Smith and S.-F. Chang. " Tools and techniques for color image retrieval", In Symposium on Electronic Imaging: Science and Technology - Storage \& Retrieval for Image and Video Databases IV, volume 2670, San Jose, CA, February 1996. IS\&T/SPIE. 
[7] J. G. Daugman, "Complete discrete 2D Gabor transforms by neural networks for image analysis and compression," IEEE Trans. ASSP, vol. 36, pp. 1169-1179, July 1998.

[8] A. K. Jain, and F. Farroknia, "Unsupervised texture segmentation using Gabor filters," Pattern Recognition, Vo.24, No.12, pp. 1167-1186, 1991.

[9] C. S. Sastry, M. Ravindranath, A. K. Pujari \& B. L. Deekshatulu, " A modified Gabor function for content based image retrieval",Pattern Recognition Letters 28 , 2007, pp 293-300.
[10] Thomas M. Deserno, Sameer Antani, and Rodney Long, 2007, "Exploring access to scientific literature using content-based image retrieval", Proc. of SPIE Vol. 6516, 65160L, pp. 65160L-1 to $65160 \mathrm{~L}-8$.

[11] Henning Muller, W. Muller, D.M.Squire, S. Maillet and T. Pun, "Performance evaluation in content based image retrieval: overview and proposals", Pattern Recognition Letters 22 (2001), pp 593-601.

[12] Manjunath B. S., Ma Y. S., 1996, “ Texture features for browsing and retrieval of image data", IEEE Trans. Pattern Anal. Machine Intell 18(8), 837-842. 